\title{
Actuator Placement for Structural Controllability beyond Strong Connectivity and towards Robustness
}

\author{
Baiwei Guo, Orcun Karaca, Sepide Azhdari, Maryam Kamgarpour, Giancarlo Ferrari-Trecate
}

April 13, 2021

\begin{abstract}
Actuator placement is a fundamental problem in control design for large-scale networks. In this paper, we study the problem of finding a set of actuator positions by minimizing a given metric, while satisfying a structural controllability requirement and a constraint on the number of actuators. We first extend the classical forward greedy algorithm for applications to graphs that are not necessarily strongly connected. We then improve this greedy algorithm by extending its horizon. This is done by evaluating the actuator position set expansions at the further steps of the classical greedy algorithm. We prove that this new method attains a better performance, when this evaluation considers the final actuator position set. Moreover, we study the problem of minimal backup placements. The goal is to ensure that the system stays structurally controllable even when any of the selected actuators goes offline, with minimum number of backup actuators. We show that this problem is equivalent to the well-studied NP-hard hitting set problem. Our results are verified by a numerical case study.
\end{abstract}

\section{Introduction}

The steady progress in computation and communication technologies is enabling the deployment of large networks of systems, which require optimal and robust coordination. Prominent examples include power grids [1] and industrial control systems [2]. A significant amount of research is currently focusing on the control design for such networks in order to achieve better performance and security 3, 4]. A fundamental design problem concerns actuator placement which aims to select a subset from all possible actuator positions to place actuators such that a chosen network metric is minimized.

Typical metrics are set functions that map the actuator positions to the costs for completing certain tasks. In [1], the authors study controllability metrics to evaluate the energy required to bring the system to an arbitrary state. Whereas, variants of the LQG cost are considered in [5]. Since some of these metrics are neither submodular nor supermodular [6], it is in general NP-hard

${ }^{*}$ The work of B. Guo and G. Ferrari-Trecate received support from the Swiss National Science Foundation under the NCCR Automation (grant agreement 51NF40_180545).

B. Guo, S. Azhdari and G. Ferrari-Trecate are with Automatic Control Laboratory, Institute of Mechanical Engineering, École Polytechnique Fédérale de Lausanne, Switzerland, e-mails: \{baiwei.guo, sepide.azhdari, giancarlo.ferraritrecate\}@epfl.ch

O. Karaca is with ABB Corporate Research Center, Baden, Switzerland. email: orcun.karaca@ch.abb.com

M. Kamgarpour is with the Electrical and Computer Eng. at the University of British Columbia, Canada. email: maryamk@ece.ubc.ca 
to find the optimal set of actuators positions [7]. ${ }^{1}$

The sets minimizing these aforementioned metrics are not guaranteed to make the resulting system controllable, considering that either the metrics are not related to controllability objective at all, or even when they are related, they involve some approximation techniques [12, 13]. In view of this issue, structurally controllable systems constitute a desirable class. These systems are the ones that attain controllability after a slight perturbation of the system parameters corresponding to edge weights in the underlying network graph [14,15]. The concept of structural controllability, along with related variants [16, 17], is based only on the graphical interconnection structure of the dynamical system and the actuator positions. The works [18] and [19 study the minimal number of input nodes under the constraint of structural controllability. Another related research direction studies this problem with the same constraint but beyond modular metrics. The work in [20] considers leader selection to minimize control errors due to noisy communication links, whereas [12] places actuators to reduce the approximate control energy metric discussed in [7]. However, both studies assume strongly connected network graphs. In this case the corresponding problem can be formulated as a matroid optimization, allowing the use of efficient greedy heuristics. Hence, the first goal of this paper is to extend the non-modular metric minimization problem under structural controllability constraints to arbitrary graphs.

In [12, 20], Forward Greedy algorithm (FG) iteratively adds the most beneficial node to the leader or the actuator position sets under the constraints of structural controllability and a cardinality upper bound. This algorithm provides a solution that approximately minimizes the given metrics. Although there exist performance guarantees for the metric value under the actuator position set returned by FG [8, 12], this algorithm does not always generate satisfactory results [21, 22]. To overcome this problem, several variants have been investigated to improve the performance, including the Continuous Greedy Algorithm [23] and the Randomized Greedy Algorithm 24]. These methods can potentially enjoy better cost upperbounds than FG in a probabilistic manner, however, they do not offer any ex-post performance improvement. Thus, the second goal of this paper is to derive a heuristic algorithm which is guaranteed to perform at least as good as FG (if not, better), while maintaining its polynomial complexity.

Another issue in actuator placement is related to susceptibility of the actuators to faults. In extreme conditions, the actuators may go offline and thus may not be able to achieve the expected performance. Related research is focused on robust optimization for the worst-case scenario [25, 26], efficient methods for contingency analysis [27], and security indices for protection of vulnerable actuators [28]. To the best of our knowledge, no previous work considers maintaining structural observability/controllability in case of offline sensor/actuators. Hence, in this paper, the third goal is to study methods to deploy backup actuators to enhance robustness of the network for structural controllability.

Targeting the aforementioned goals, our contributions are as follow. First, in case of a network graph which is not necessarily strongly connected, we propose a method to select actuators and, under certain conditions, we guarantee the structural controllability of the resulting system. Second, based on FG, we propose a novel method, called Long-Horizon Forward Greedy Algorithm (LHFG). The performance of the actuator position set derived is guaranteed to be at least as good as (if not, better than) that of FG. Third, we formulate the problem of minimal backup placements. The goal is to find the minimal backup actuator set such that the system can maintain its structural controllability even when any of the selected actuators goes offline. We show that this problem is equivalent to the hitting set problem.

\footnotetext{
${ }^{1}$ Many works concerning such nonmodular objectives instead consider approximate submodularity or supermodularity properties defined by certain ratios, see for instance 8 11.
} 
The remainder of this paper is organized as follows. In Section 2, we introduce the problem formulation and preliminaries. In Section 3, we provide an extension of actuator placement to arbitrary graphs and we present LHFG, which is shown to provide an improvement over FG. In Section 4, we propose an efficient scheme for finding positions to place backups. To verify our results, we present a numerical case study in Section 5. Some conclusions are provided in Section 6 .

Remarks on notation: For notational simplicity, we use $v$ and $\{v\}$ interchangeably for singleton sets. Within pseudocodes of algorithms, we introduce several symbols for sets and variables to describe the steps of the algorithms. These notations, without being defined in the main body of this paper, will be used in the proofs.

\section{Problem formulations and preliminaries}

\subsection{Problem formulations}

Consider a linear system with state vector $x \in \mathbb{R}^{n}$. To each state variable $x_{i} \in \mathbb{R}$, we associate a node $v_{i} \in V:=\left\{v_{1}, \ldots, v_{n}\right\}$. If we place actuators on the set of nodes $S \subset V$, called the actuator position set, and apply an input vector $u \in \mathbb{R}^{n}$, the system dynamics can be written as

$$
\dot{x}=A x+B(S) u,
$$

where $B(S)=\operatorname{diag}(\mathbf{1}(S)) \in \mathbb{R}^{n \times n}$ and $\mathbf{1}(S)$ denotes a vector of size $n$ whose $i$ th entry is 1 if $v_{i}$ belongs to $S$ and 0 otherwise. Let $G=(V, E)$ denote a weighted directed graph associated with the adjacency matrix $A$ with nodes $V$ and edges $E$, where $|E|=l$ and the directed edge $\left(v_{j}, v_{i}\right) \in E$ if the associated weight $(A)_{i j}$ is non-zero.

The pair $(A, B(S))$ is called controllable if for all $x_{0}, x_{1} \in \mathbb{R}^{n}$ and $T>0$ there exists a control input $u:[0, T] \rightarrow \mathbb{R}^{n}$ that steers the system from $x_{0}$ at $t=0$ to $x_{1}$ at $t=T$. For linear time-invariant systems, controllability can be verified by the rank of the controllability matrix $P=\left[\begin{array}{llll}B(S) & A B(S) & \cdots & A^{n-1} B(S)\end{array}\right] \in \mathbb{R}^{n \times n^{2}}$. Due to potential errors in the identification of the edge weights, most of the time we can only rely on the topology but not on the particular weights. Motivated by this particularity, we introduce the weaker notion of structural controllability.

Definition 1 We say that $(A, B)$ and $(\hat{A}, \hat{B})$ with $A, B, \hat{A}, \hat{B} \in \mathbb{R}^{n \times n}$ have the same structure if matrices $[A B]$ and $[\hat{A} \hat{B}]$ have zeros at the same entries. Given $S \subset V,(A, B(S))$ is structurally controllable if there exists a controllable pair $(\hat{A}, \hat{B})$ having the same structure as $(A, B(S))$.

As shown in [14], structural controllability of the pair $(A, B)$ further implies that even when $(A, B)$ is not controllable, it is always possible to slightly perturb some non-zero edge weights to ensure controllability.

With this notion, the first problem we aim to solve is the following.

P1: Suppose a potentially non-modular and non-increasing metric $f: 2^{V} \rightarrow \mathbb{R}$ is given. Find $K$ actuators attaining structural controllability while minimizing the metric at hand:

$$
\begin{array}{cl}
\min _{S \subset V,|S|=K} & f(S) \\
\text { s.t. } & (A, B(S)) \text { is structurally controllable. }
\end{array}
$$

Suppose $S$ is a feasible solution to $\mathbf{P} \mathbf{1}$, which could be either optimal or approximately optimal. The second problem considers the robustness of $(A, B(S))$ against failures, which will be further motivated in Section 4 . 
P2: Given $S$, find the minimal backup actuator positions such that structural controllability can be retained when any single actuator at $v \in S$ malfunctions:

$$
\begin{array}{cl}
\min _{\mathcal{B} \subset V} & |\mathcal{B}| \\
\text { s.t. } & \forall v \in S, \exists b_{v} \in \mathcal{B},\left(A, B\left(\{S \backslash v\} \cup b_{v}\right)\right) \text { is } \\
& \text { structurally controllable. }
\end{array}
$$

To define the constraints on these two problems, we need an efficient characterization of structural controllability.

\subsection{Characterization of structural controllability}

Structural controllabilty boils down to checking two graphical properties. A system $(A, B(S))$ is structurally controllable if and only if it satisfies accessibility and dilation-freeness [14, Theorem 1], which are defined as follow.

Definition 2 The system $(A, B(S)$ ) satisfies accessibility (or the nodes in $V$ are accessible by $S$ ) if for any $v \in V$ there exists a path from a node in $S$ to $v$ in $G=(V, E)$.

Note that one can use Breadth First Search to verify accessibility condition. For dilationfreeness, on the other hand, we need a method to distinguish a node with an actuator from other nodes in the graph $G=(V, E)$. Let $S^{\prime \prime}=\left\{v_{s_{1}}^{\prime \prime}, \ldots, v_{s_{K}}^{\prime \prime}\right\}$ denote a copy of the actuator position set $S=\left\{v_{s_{1}}, \ldots, v_{s_{K}}\right\}$ and $E^{\prime \prime}$ denote edges connecting $v_{s_{i}}^{\prime \prime}$ to $v_{s_{i}}, i=1, \ldots, K$. Then, the graph $G^{\prime \prime}=\left(V \cup S^{\prime \prime}, E \cup E^{\prime \prime}\right)$ illustrates explicitly how the actuators are connected to the nodes in $V$.

Definition 3 The system $(A, B(S))$ is dilation-free if in $G^{\prime \prime}$ the in-neighbor set $N(U):=\left\{\tilde{v} \mid \exists v_{u} \in\right.$ $V \cup S^{\prime \prime}$ s.t. $\left.\left(\tilde{v}, v_{u}\right) \in E \cup E^{\prime \prime}\right\}$ for any subset $U \subset V$ satisfies $|N(U)| \geq|U|$.

Dilation-freeness requirement for controllability can be interpreted as follows. Whenever there are fewer actuators connected to a set of nodes than the cardinality of the set under consideration, we do not possess the flexibility to steer the states of these nodes arbitrarily to achieve any controllability notion.

In the following, we introduce methods for dilation-freeness checks via matchings in bipartite graphs 29,30$]$.

We define concepts related to bipartite graphs. An undirected graph is called bipartite and denoted as $\left(V^{1}, V^{2}, \mathrm{E}\right)$ if its vertices are partitioned into $V^{1}$ and $V^{2}$, while any undirected edge in $\mathrm{E}$ connects a vertex in $V^{1}$ to another in $V^{2}$. A matching $m$ is a subset of $\mathrm{E}$ where no two edges in $m$ share a vertex in common. Given a subset $L \subset V^{1} \cup V^{2}$, we say $L$ is covered by $m$ if any $v \in L$ is incident to an edge in $m$. The matching $m$ is called maximum if it has the largest cardinality among all possible matchings and is called perfect if $V^{2}$ is covered.

We utilize an auxiliary bipartite graph to check dilation-freeness. It is constructed as follows. Let node sets $V^{\prime}=\left\{v_{1}^{\prime}, \ldots, v_{n}^{\prime}\right\}$ and $V^{\prime \prime}=\left\{v_{1}^{\prime \prime}, \ldots, v_{n}^{\prime \prime}\right\}$ be two copies of $V=\left\{v_{1}, \ldots, v_{n}\right\}$. As for the edges, the set $\mathrm{E}$ consists of undirected edges connecting $v_{i}$ with $v_{j}^{\prime}$ if $\left(v_{i}, v_{j}\right) \in E$, whereas the edge set $\mathrm{E}_{S}$ consists of undirected edges connecting $v_{k}^{\prime}$ with $v_{k}^{\prime \prime}$ if $v_{k} \in S$. The auxiliary bipartite graph is then given by $\mathcal{H}_{b}(S):=\left(V \cup S^{\prime \prime}, V^{\prime}, \mathrm{E} \cup \mathrm{E}_{S}\right)$. With this graph at hand, the following lemma [12, Proposition 7] provides an efficient method to check dilation-freeness.

Lemma 1 (Dilation-freeness check) Let $\mathcal{C}_{K}=\{S \subset V|| S \mid=K$ and the pair $(A, B(S))$ is dilation-free $\}$ and $\tilde{\mathcal{C}}_{K}=\left\{\Omega \mid \exists S \in \mathcal{C}_{K}\right.$ such that $\left.\Omega \subset S\right\}$. With $\bar{m}(S)$ as a maximum matching in $\mathcal{H}_{b}(S)$, we have 


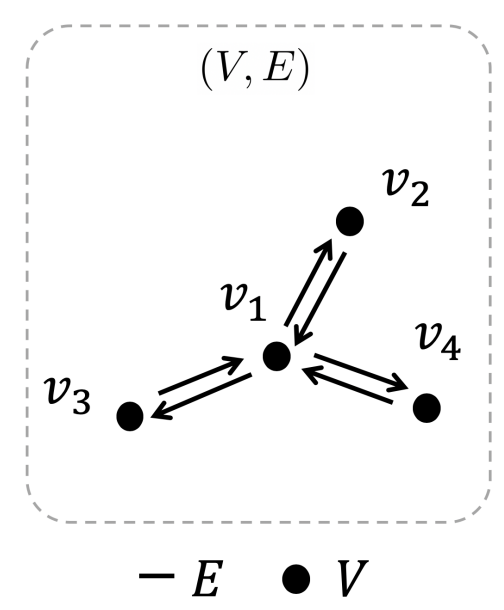

Figure 1: Graph for the 4-node system

(i) $S \in \mathcal{C}_{K}$ if and only if $|S|=K$ and $\bar{m}(S)=n$,

(ii) $S \in \tilde{\mathcal{C}}_{K}$ if and only if $|S| \leq K$ and $\bar{m}(S) \geq n-K+|S|$.

Note that a maximum matching can easily be derived via Edmonds-Karp Algorithm with the complexity of $O\left(n l^{2}\right) 31$. We briefly discuss the motivation for defining $\tilde{\mathcal{C}}_{K}$, a family of sets, and checking membership to it. The set $\tilde{\mathcal{C}}_{K}$ contains all the sets that can be expanded to a set $S$ such that $|S|=K$ and $(A, B(S))$ is dilation-free. Checking membership with respect to $\tilde{\mathcal{C}}_{K}$ is essential to implementing the greedy algorithm to approximately solve $\mathbf{P} \mathbf{1}$, a popular polynomial-time heuristic in the literature to iteratively solve combinatorial optimization problems [32]. This method will be discussed in detail in Section 2.3 .

We illustrate these dilation-freeness checks with the following example.

Example 1 Consider a system described by 4 nodes and the dynamic equations (1) where

$$
A=\left[\begin{array}{cccc}
0 & -0.5 & -0.8 & -0.6 \\
1 & 0 & 0 & 0 \\
1 & 0 & 0 & 0 \\
1 & 0 & 0 & 0
\end{array}\right]
$$

The graph $G=(V, E)$ corresponding to the adjacency matrix $A$ is provided in Figure 1 . From the bipartite $\mathcal{H}_{b}(\emptyset)$ in Figure 2, we see that any maximum matching consists of 2 edges. Only if $K \geq 2$ do we have that $\bar{m}(\emptyset) \geq n-K+|\emptyset|$ and $\emptyset \in \tilde{\mathcal{C}}_{K}$, which is to say $\tilde{\mathcal{C}}_{K} \neq \emptyset$. Thus, $K=2$ is the minimum number of actuators required for dilation-freeness. Then we check the actuator set $S=\left\{v_{3}, v_{4}\right\}$. The bipartite graph $\mathcal{H}_{b}(S)$ is shown in Figure 2, from which we see the maximum matching contains 4 edges and thus $S \in \mathcal{C}_{2}$.

\subsection{Forward greedy algorithm for structural controllability}

A special instance of $\mathbf{P 1}$ has been studied in [12, 20], where strong connectivity of $G$, the directed graph corresponding to the adjacency matrix $A$, is assumed. With this assumption, $(A, B(S))$ satisfies accessibility whenever $|S|>0$. Consequently, if $|S| \neq 0$, dilation-freeness implies structural controllability. $\mathbf{P} 1$ is then reduced to minimization of $f(S)$ with the constraint $S \in \tilde{\mathcal{C}}_{K}$, which is a matroid optimization problem [12]. 

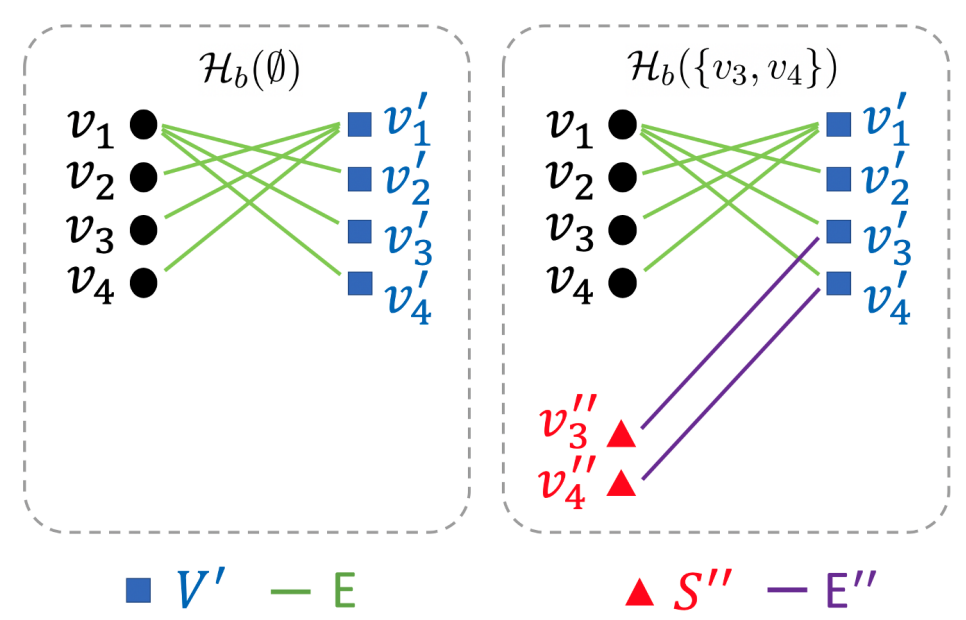

Figure 2: Auxiliary bipartite graphs $\mathcal{H}_{b}(\emptyset)$ and $\mathcal{H}_{b}\left(\left\{v_{3}, v_{4}\right\}\right)$

The study in [12] proposes to apply the Forward Greedy Algorithm (FG) through the function $\mathrm{FG}\left(S^{0}, d\right)$ shown in Algorithm 1. Among the inputs, $S^{0}$ denotes the initial set before expansion and $d$, called the depth, denotes an upper bound for the number of expansions. In the pseudocode of Algorithm 1, the function ISMEMBER is implemented using the efficient graph-theoretical techniques introduced in Section 2.2. This algorithm can be justified by the following.

Fact ( $[\mathbf{1 2}]$ ) If $G$ is strongly connected, with $d=K-\left|S_{0}\right|$ and $S^{0} \in \tilde{\mathcal{C}}_{K}, S_{\mathrm{f}}=\mathrm{FG}\left(S^{0}\right.$,d) satisfies that $S_{\mathrm{f}} \in \mathcal{C}_{K}$.

In addition to the fact above, one can utilize special properties of matroids 33 and provide a suboptimality bound on $f\left(S_{\mathrm{f}}=\mathrm{FG}(\emptyset, K)\right)$ with respect to $f\left(S^{*}\right)$, given the submodularity ratio and the curvature of the metric $f[22]$. However, these results rely on the strong connectivity of $G$. If the digraph $G$ is not strongly connected, dilation-freeness of $(A, B(S))$ does not necessarily lead to structural controllability. In view of this, the greedy solution may fail to attain structural controllability.

In Section 3 below, targeting P1, we 1) extend FG beyond the assumption of strong connectivity by providing an efficient method to attain the accessibility condition and 2) propose a novel algorithm based on FG with a longer horizon and ensuring a better performance. In Section 4 , we address the solution to $\mathbf{P 2}$.

\section{Extensions to the greedy heuristics}

\subsection{Beyond strong connectivity}

In case the graph $G=(V, E)$ is not strongly connected, we consider finding an initial set $S_{0}$ before running FG such that all the nodes in $V$ are accessible by $S_{0}$.

We start by highlighting that the well-establish Kosaraju's algorithm [29] can construct all the strongly connected components in the directed graph $G$ in linear time, that is, with complexity $O(n+l)$, where $l$ is the number of edges in $G$. Whenever $G$ is not strongly connected, there would be components with no incoming edges and we denote them as $\mathcal{T}^{1}, \ldots, \mathcal{T}^{n \mathcal{T}}$. If all the nodes in $V$ are accessible by a set $S$, then for any $1 \leq i \leq n_{\mathcal{T}}$ the component $\mathcal{T}^{i}$ guarantees that $\mathcal{T}^{i} \cap S \neq \emptyset$, otherwise the nodes in $\mathcal{T}^{i}$ would not be accessible. Based on this idea, we propose Algorithm 2 to 

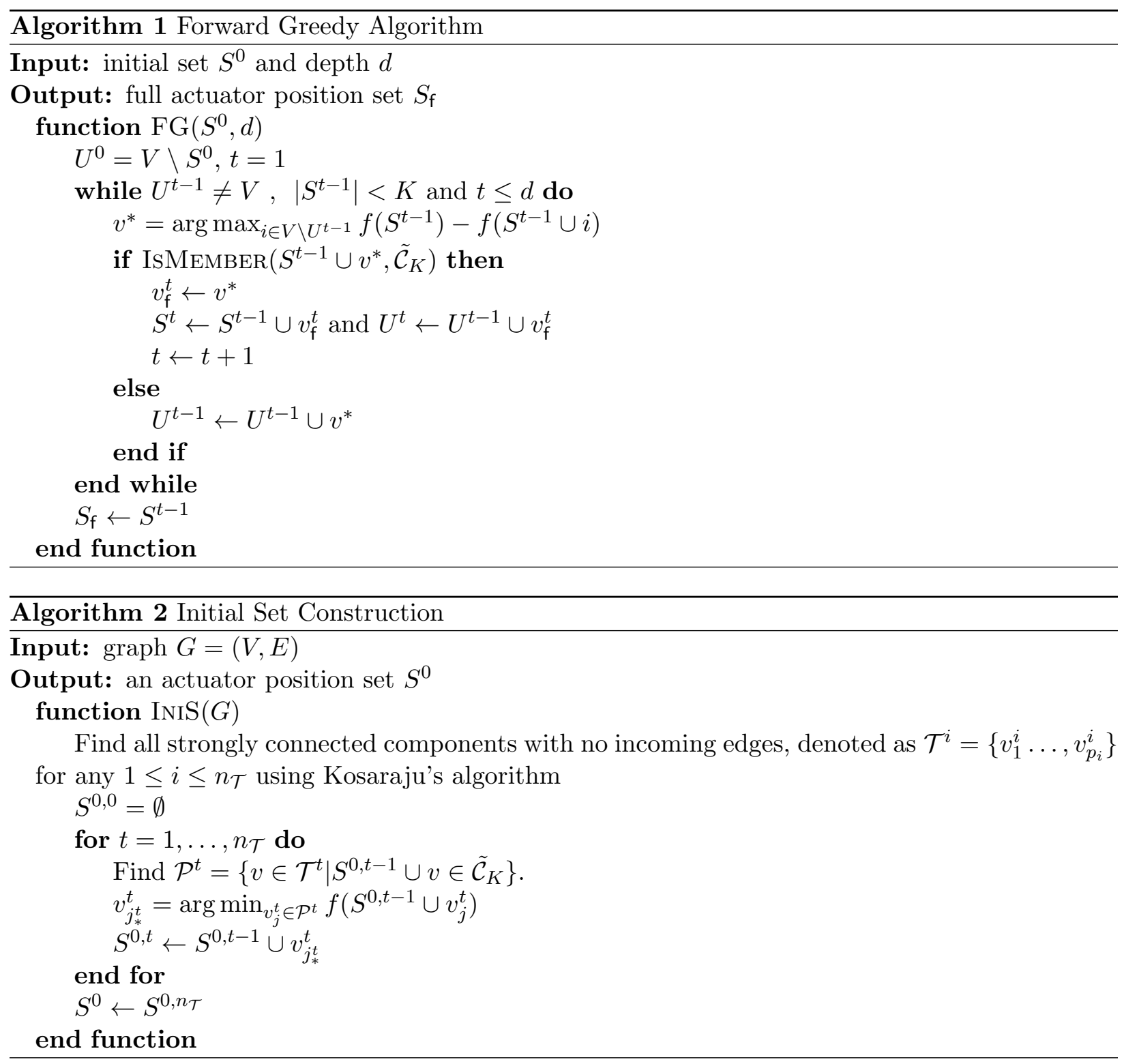

construct $S^{0}$ when $G$ is not strongly connected.

The following proposition proves that under certain conditions one can use Algorithm 1 to further expand $S^{0}$ such that the derived system is structurally controllable.

Proposition 1 Let $k$ be the smallest integer such that $\mathcal{C}_{k} \neq \emptyset$. If $n_{\mathcal{T}}$ satisfies $n>K \geq k+n_{\mathcal{T}}$, then

(i) the set $S^{0}$ derived through Algorithm 2 belongs to $\tilde{\mathcal{C}}_{K}$,

(ii) By applying Algorithm 1 to further expand $S^{0}$ and obtaining $S_{\mathrm{f}}=\mathrm{FG}\left(S^{0}, K-\left|S^{0}\right|\right)$, the resulting system $\left(A, B\left(S_{\mathrm{f}}\right)\right)$ is structurally controllable.

Proof For the proof of (i), for any $t$, we verify that $v_{j_{*}^{t}}^{t}$ is well defined and we then show that $S^{0, t} \in \tilde{\mathcal{C}}_{K}$. 


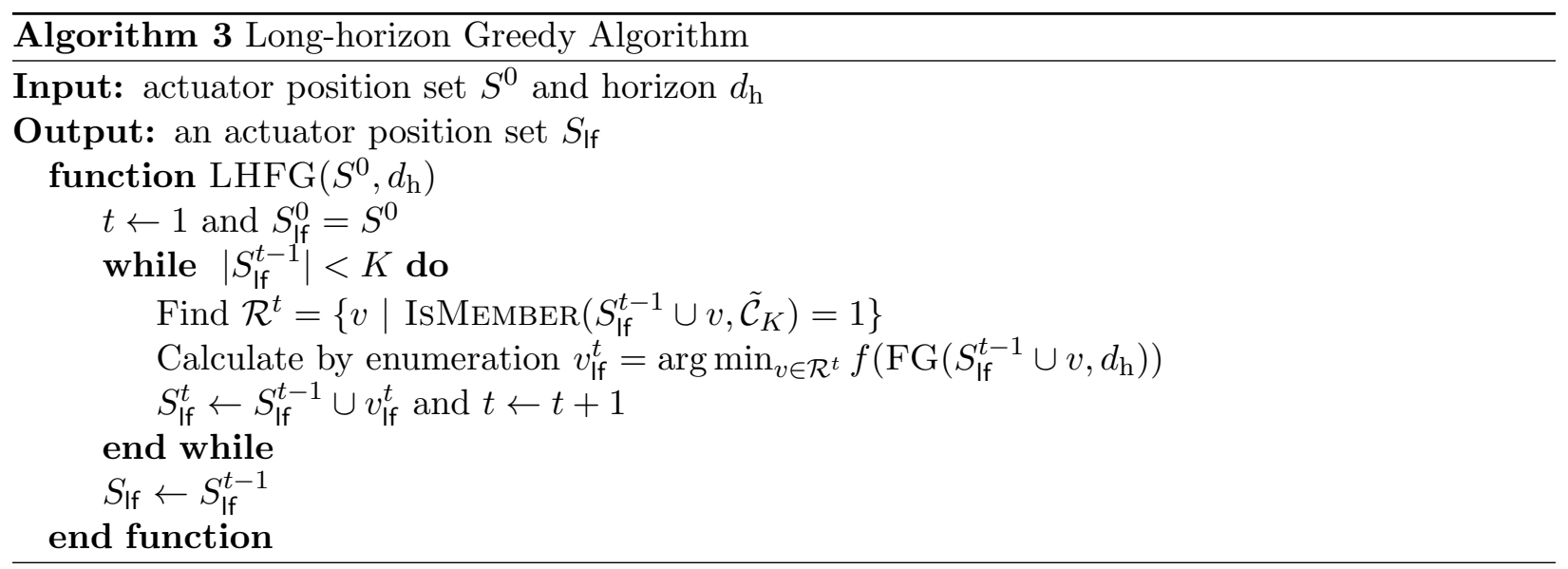

Let $W$ be a set in $\mathcal{C}_{k}$ and notice that for any $S \subset V$ the system $(A, B(W \cup S))$ is dilation-free. In Algorithm 2, since for $1 \leq t \leq n_{\mathcal{T}}$ and any $v \in V$ we have $\left|S^{0, t-1} \cup v\right| \leq n_{\mathcal{T}}, S^{0, t-1} \cup v \cup W \in \tilde{\mathcal{C}}_{K}$ and thus $\mathcal{P}^{t} \neq \emptyset$, which means for each $t$ we can derive $v_{j_{*}^{t}}^{t} \in \mathcal{P}^{t}$. Suppose $S^{0, t-1} \in \tilde{\mathcal{C}}_{K}$, then $S^{0, t}=S^{0, t-1} \cup v_{j_{*}^{t}}^{t} \in \tilde{\mathcal{C}}_{K}$. By induction, $S^{0} \in \tilde{\mathcal{C}}_{K}$.

Regarding the statement (ii), to prove the structural controllability of $\left(A, B\left(S_{\mathrm{f}}\right)\right)$, we need to show this system satisfies both accessibility and dilation-freeness.

Since for any $t v_{j_{*}^{t}}^{t} \in \mathcal{T}^{t}, S^{0} \cap \mathcal{T}^{t} \neq \emptyset$. From graph theory, any node $v \in V$ is accessible by $S^{0} \subset S_{\mathrm{f}}$. The method to prove dilation-freeness follows closely that of " $S^{0, t} \in \tilde{\mathcal{C}}_{K}$ " provided above, and thus omitted.

In case a large number of actuators is allowed, the condition " $n>K \geq k+n_{\mathcal{T}}$ " is not restrictive. Whenever this condition does not hold, we may still obtain $S^{0} \in \tilde{\mathcal{C}}_{K}$, and we can then use FG to expand $S^{0}$.

To summarize, in case $G$ is not strongly connected, our overall method for actuator placement is to first run Algorithm 2 to obtain the initial set $S^{0}=\operatorname{InIS}(G)$ and then to execute Algorithm 1 to obtain the actuator position set $S_{\mathrm{f}}=\mathrm{FG}\left(S^{0}, K-\left|S^{0}\right|\right)$. Since this procedure is not the standard greedy algorithm, the upper bounds for the suboptimality gaps in previous works are not applicable. We leave deriving a guarantee towards the performance of the actuator position set $\mathrm{FG}\left(\operatorname{InIS}(G), K-\left|S_{0}\right|\right)$ as a future work.

\subsection{Beyond myopic decisions}

This section derives a method that improves upon FG in terms of the performance of the final actuator position set.

For the iterations in Algorithm 1, the marginally most beneficial nodes, $v_{\mathrm{f}}^{1}, \ldots, v_{\mathrm{f}}^{K-\left|S^{0}\right|}$, are added one after the other. These decisions are myopic, since a node added at some iteration might make it harder to further decrease the given metric in later iterations. To mitigate this issue, we equip FG with a long horizon, as shown in Algorithm 3. This way, we can evaluate whether to add a node based on how the addition of this node influences the actuator position set expansion in the future.

We call this new method Long-Horizon Greedy Algorithm (LHFG). We achieve the longer horizon by using FG to further expand starting from the node we are considering. The depth of this embedded FG is called the horizon of LHFG. 
The following proposition proves that with a horizon long enough, LHFG has a performance no worse than FG.

Proposition 2 For an arbitrary network, if $S^{0} \in \tilde{\mathcal{C}}_{K}, f\left(S_{\text {If }}\right) \leq f\left(S_{\mathrm{f}}\right)$, where $S_{\text {If }}=\operatorname{LHFG}\left(S^{0}, K-\right.$ $\left.\left|S^{0}\right|\right)$ and $S_{\mathrm{f}}=\mathrm{FG}\left(S^{0}, K-\left|S^{0}\right|\right)$.

Proof Following the proof of Proposition 1 regarding dilation-freeness, one can see that $S_{\mathrm{If}}$ and $S_{\mathrm{f}}$ are both well-defined and belong to the set $\mathcal{C}_{K}$. Next, we show $S_{\text {If }}$ achieves a lower metric value.

By denoting $\bar{S}_{\mathrm{lf}}^{t}:=\mathrm{FG}\left(S_{\mathrm{lf}}^{t}, K-\left|S^{0}\right|\right)$, we claim that $f\left(\bar{S}_{\mathrm{lf}}^{t}\right) \geq f\left(\bar{S}_{\mathrm{lf}}^{t+1}\right)$ for $t<K-\left|S_{0}\right|$. To prove this, we notice that $\bar{S}_{\mathrm{If}}^{t} \backslash S_{\mathrm{If}}^{t} \subset \mathcal{R}^{t+1}$ and there exists $\tilde{v} \in \bar{S}_{\mathrm{If}}^{t} \backslash S_{\mathrm{If}}^{t}$ such that $\tilde{v} \in \mathcal{R}^{t+1}$ and $\bar{S}_{\text {lf }}^{t}=\mathrm{FG}\left(S_{\mathrm{lf}}^{t} \cup \tilde{v}, K-\left|S^{0}\right|\right)$, therefore, $f\left(\bar{S}_{\mathrm{lf}}^{t+1}\right)=\arg \min _{v \in \mathcal{R}^{t+1}} f\left(\mathrm{FG}\left(S_{\mathrm{lf}}^{t} \cup v, K-\left|S^{0}\right|\right)\right) \leq$ $f\left(\mathrm{FG}\left(S_{\mathrm{lf}}^{t} \cup \tilde{v}, K-\left|S^{0}\right|\right)\right)=f\left(\bar{S}_{\mathrm{lf}}^{t}\right)$.

To proceed, we recall that $v_{\mathrm{lf}}^{1}, \ldots, v_{\mathrm{lf}}^{K-\left|S_{0}\right|}$, in order, are added to form $S_{\mathrm{lf}}$ while $v_{\mathrm{f}}^{1}, \ldots, v_{\mathrm{f}}^{K-\left|S_{0}\right|}$, in order, are added to form $S_{\mathrm{f}}$. If $i$ is the smallest integer such that $v_{\mathrm{lf}}^{i} \neq v_{\mathrm{f}}^{i}, f\left(S_{\mathrm{f}}\right)=f\left(\bar{S}_{\mathrm{lf}}^{i-1}\right) \geq$ $f\left(\mathrm{FG}\left(S_{\mathrm{lf}}^{i-1} \cup v_{\mathrm{lf}}^{i}, K-\left|S^{0}\right|\right)\right)=f\left(\bar{S}_{\mathrm{lf}}^{i}\right) \geq \bar{S}_{\mathrm{lf}}^{K-\left|S^{0}\right|}$. By noticing that $S_{\mathrm{lf}}=\bar{S}_{\mathrm{lf}}^{K-\left|S^{0}\right|}$, we have $f\left(S_{\mathrm{lf}}\right) \leq$ $f\left(S_{\mathrm{f}}\right)$.

We cannot guarantee that $f\left(S_{\text {If }}\right)<f\left(S_{\mathrm{f}}\right)$ because of two potential cases, i) $S_{\text {If }}=S_{\mathrm{f}}$ and ii) $f\left(S_{\mathrm{lf}}\right)=f\left(S_{\mathrm{f}}\right)$ even if $S_{\mathrm{If}} \neq S_{\mathrm{f}}$. However, in practice, we expect these two cases to be rare. We refer the readers to Section 5 for a numerical case study where $f\left(S_{\text {If }}\right)$ is significantly smaller than $f\left(S_{\mathrm{f}}\right)$.

Notice that, to derive $S_{\text {If }}=\operatorname{LHFG}\left(\operatorname{InIS}(G), K-\left|S_{0}\right|\right)$, most of the computational time would be spent on long horizon evaluations. During these evaluations, we execute FG for at most $K(2 n-$ $K+1) / 2$ times. Suppose the complexity of calculating $f(S)$ is $O(q(n))$. Note that the complexity of ISMEMBER is $O\left(n l^{2}\right)$ from Edmonds-Karp algorithm. One can then verify that the complexity of LHFG is $O\left(K n^{3}\left(q(n)+l^{2}\right)\right)$. To reduce the computational complexity, one can shorten the horizon $d_{\mathrm{h}}$ of LHFG. However, it is then not possible to guarantee better performance than $\mathbf{F G}$, as in the proposition above. In Section 5, we study the computational time and the derived actuator sets in a numerical example for $d_{\mathrm{h}}<K-\left|S_{0}\right|$.

\section{Backup placements for ensuring structural controllability in re- sponse to failures}

In many applications, the selected actuators $S$, that we call primary, derived through LHFG may be offline due to potential damages/failure. An offline primary actuator can potentially make the system uncontrollable, which would be unacceptable. In this section, we first list two assumptions that motivate P2. We then show that $\mathbf{P 2}$ is equivalent to hitting set problem, which has been well studied in the combinatorial optimization literature.

Assumption 1 Only one actuator at a time can be offline.

This assumption can hold if primary actuator failures are not frequent and/or offline actuators can be restored quickly. If several actuators can go offline at the same time, at the current stage of our research, we need to enumerate all possible combinations of failures and it would be quite conservative to deploy backups for the worst case scenario.

Assumption 2 There exists at least one actuator $v$ such that $(A, B(S \backslash v))$ is not structurally controllable. 
A primary actuator is called essential if its failure violates the structural controllability. In practice, even if the number of primary actuators is more than the minimum needed for structural controllability, it is often the case that the assumption above holds. We illustrate this phenomenon in Section 5 with a numerical example.

Due to Assumption 2, it is necessary to have backups for essential primary actuators. Under these assumption, $\mathbf{P 2}$ in Section 2.1 provides us with the minimal backup position set $\mathcal{B}$. With these backup actuators of $\mathcal{B}$, we can still retain structural controllability by replacing any single offline primary actuator.

To solve P2, we need to characterize its constraint set in a tractable manner. For this purpose, following definitions are in order.

Definition 4 Given the actuator position set $S$ such that $(A, B(S))$ is structurally controllable, we say $v$ is a DFR (dilation-freeness-recovering) backup position for $v_{\text {off }} \in S$ if $\left(A, B\left(S \backslash v_{\text {off }} \cup v\right)\right)$ is dilation-free. We say $v$ is a feasible backup position for $v_{\text {off }}$ if $\left(A, B\left(S \backslash v_{\text {off }} \cup v\right)\right)$ is structurally controllable.

We will now provide a tractable characterization of the feasible backup positions for a given $v_{\text {off }}$. To start with, consider the DFR backup positions. Recall that $S \in \mathcal{C}_{K}$ if and only if there exists a perfect matching $m_{0}$ in the bipartite graph $\mathcal{H}_{b}(S)$. With the primary actuator at $v_{\text {off }}$ going offline, checking whether node $v$ is a DFR backup position is equivalent to checking whether there exists a perfect matching in $\mathcal{H}_{b}\left(S \backslash v_{\text {off }} \cup v\right)$. For any $v \in V$, one can run the Edmonds-Karp Algorithm on $\mathcal{H}_{b}\left(S \backslash v_{\text {off }} \cup v\right)$. By iteratively doing so, we can obtain all DFR backup positions for $v_{\text {off }}$, with the complexity of $n^{2} l^{2}$. Such a naive approach fails to exploit the properties of matchings in bipartite graphs.

The following theorem characterizes all the DFR backup positions for $v_{\text {off }}$ in a computationally efficient way.

Theorem 1 Let $S \in \mathcal{C}_{K}$ be the set of actuators. Suppose $v_{\mathrm{off}} \in S$ is offline and dilation freeness is lost, that is, there does not exist a perfect matching in $H_{1}:=\mathcal{H}_{b}\left(S \backslash v_{\text {off }}\right) . \quad A$ node $v \neq v_{\text {off }}$ is DFR for $v_{\text {off }}$ if and only if there exist a perfect matching $m_{0}$ in $\mathcal{H}_{b}(S)$ and an alternating path $p=\left(v_{\text {off }}^{\prime}, v_{p_{1}}, v_{p_{2}}^{\prime}, \ldots, v_{p_{r}}, v^{\prime}\right)$ where edges $\left(v_{o f f}^{\prime}, v_{p_{1}}\right),\left(v_{p_{2}}^{\prime}, v_{p_{3}}\right), \ldots,\left(v_{p_{r-1}}^{\prime}, v_{p_{r}}\right) \notin m_{0}$, $\left(v_{p_{1}}, v_{p_{2}}^{\prime}\right), \ldots,\left(v_{p_{r}}, v^{\prime}\right) \in m_{0}$.

Proof For both parts, observe that since there does not exist a perfect matching in $\mathcal{H}_{b}\left(S \backslash v_{\text {off }}\right)$, the matching $m_{0}$ must contain the edge $\left(v_{\text {off }}^{\prime \prime}, v_{\text {off }}^{\prime}\right)$.

Sufficiency: By utilizing the alternating path $p$, we exclude from $m_{0}$ the edges $\left(v_{\text {off }}^{\prime \prime}, v_{\text {off }}^{\prime}\right)$, $\left(v_{p_{1}}, v_{p_{2}}^{\prime}\right), \ldots,\left(v_{p_{r}}, v^{\prime}\right)$ and include $\left(v_{o f f}^{\prime}, v_{p_{1}}\right),\left(v_{p_{2}}^{\prime}, v_{p_{3}}\right), \ldots,\left(v_{p_{r-1}}^{\prime}, v_{p_{r}}\right),\left(v^{\prime}, v^{\prime \prime}\right)$ to form a new edge set $m_{2}$. One can verify that all the edges in $m_{2}$ are contained in $H_{2}:=\mathcal{H}_{b}\left(S \backslash v_{\text {off }} \cup v\right)$ and $m_{2}$ is a perfect matching in $\mathrm{H}_{2}$, in other words, $v$ is a DFR backup for $v_{\text {off. }}$

Necessity: Since $v$ is a DFR backup position for $v_{\text {off }}$, there exists a perfect matching in $\mathrm{H}_{2}$. In this bipartite graph with the non-perfect matching $m_{0} \backslash\left(v_{\text {off }}^{\prime \prime}, v_{\text {off }}^{\prime}\right)$, there exists an augmenting path $p_{\alpha}$ and by augmentation on this path one can obtain in $\mathrm{H}_{2}$ a perfect matching [29], denoted as $m_{\alpha}$. Due to the fact that there does not exist a perfect matching in $H_{1}$, the node $v^{\prime \prime}$ must be incident to $p_{\alpha}$. Moreover, the node $v_{\text {off }}^{\prime}$ is also incident to $p_{\alpha}$, otherwise the perfect matching $m_{\alpha}$ formed by augmentation cannot cover $v_{\text {off }}^{\prime}$ which contradicts the perfectness. We trim $p_{\alpha}$ to form $p_{\beta}$ such that $p_{\beta}$ only contains the part of $p_{\alpha}$ between $v_{\text {off }}^{\prime}$ and $v^{\prime}$. One can verify that $p_{\beta}$ is an alternating path in $\mathcal{H}_{b}(S)$ with respect to the matching $m_{0} \backslash\left(v_{\mathrm{off}}^{\prime \prime}, v_{\mathrm{off}}^{\prime}\right)$ and satisfies the characterization specified in the theorem. 
Remark If the assumptions of Theorem 1 do not hold, i.e., there exists a perfect matching in $H_{1}$, the actuator $v_{\text {off }}$ going offline does not affect the dilation-freeness property. Hence, this actuator is not essential and any node can be a DFR backup position.

To find all the alternating paths described in Theorem 1, we can use Breadth-First Search, whose complexity is $O(n+l)$. This allows us to efficiently find the DFR backup position set $\mathcal{D}(v)$, for any $v \in V$. Note that $v \in \mathcal{D}(v)$.

It is then tractable to derive the feasible backup position set $\mathcal{F}\left(v_{\text {off }}\right)$ from the DFR backup position sets. It holds that $\mathcal{F}\left(v_{\text {off }}\right)=\mathcal{D}\left(v_{\text {off }}\right)$ except for the following situation. Recall that through Algorithm 2 we derive the strongly connected components with no incoming edges $\mathcal{T}^{1}, \ldots, \mathcal{T}^{n \mathcal{T}}$. If there exists $i \in \mathbb{Z}^{+}$such that $v_{\text {off }} \in \mathcal{T}^{i}$ and $S \cap \mathcal{T}^{i}=v_{\text {off }}$, then the actuator at $v_{\text {off }}$ is the only one in $\mathcal{T}^{i}$. In case it goes offline, the system $\left(A, B\left(S \backslash v_{\text {off }}\right)\right)$ no longer satisfies accessibility. For this specific $v_{\text {off }}$, we should have $\mathcal{F}\left(v_{\text {off }}\right)=\mathcal{D}\left(v_{\text {off }}\right) \cap \mathcal{T}^{i}$.

With the feasible backup position sets $\mathcal{F}\left(v_{1}\right), \ldots, \mathcal{F}\left(v_{K}\right)$ obtained via Theorem 1, P2 can be reformulated as follows.

Corollary 1 Given the primary actuator positions $S$, the optimization problem $\mathbf{P} 2$ is equivalent to the following:

$$
\begin{aligned}
\min _{\mathcal{B} \subset V} & |\mathcal{B}| \\
\text { s.t. } & \forall v \in S, \exists b_{v} \in \mathcal{B}, \text { with } b_{v} \in \mathcal{F}(v) .
\end{aligned}
$$

This is the classical hitting set problem [34, which is well-known to be NP-hard. There are extensive studies proposing efficient approximate solutions with provable approximation ratios, e.g., the LP-based approach in [35] and the randomized algorithm in [36].

As a summary, our overall method is to first find the primary actuators using the methods in Section 3. We then construct the feasible backup position sets for the essential ones. Finally, we solve the hitting set problem.

\section{A numerical case study}

We test our algorithms on a linear system whose system matrix $A$ corresponds to the digraph $G$ illustrated in Figure $3{ }^{2}$ In this graph, the edge weights are set to be all 1 and there are 5 strongly connected components.

The metric under investigation is an approximate controllability metric $f=F_{\epsilon}$, where $F_{\epsilon}(S)=$ $\operatorname{tr}\left(\left(W_{T}(S)+\epsilon I\right)^{-1}\right)$, and $W_{T}(S)=\int_{0}^{T} e^{A \tau} B(S) B^{\top}(S) e^{A^{\top} \tau} d \tau$ is the controllability Grammian. This metric measures the average energy required for steering the system from $x_{0}$ with $\left\|x_{0}\right\|_{2}=1$ at $t=0$ to zero state at $t=T$. The constant term $\epsilon I$ allows for FG and LHFG to evaluate an uncontrollable actuator position set $S^{0} \notin \mathcal{C}_{K}$. We kindly refer the interested reader to 12 , 25 for more discussions on the metric $F_{\epsilon}(S)$. We let $\epsilon=10^{-12}$ and $T=1$. Our goal is to find an actuator set $S$ with cardinality 9 that minimizes the metric $F_{\epsilon}(S)$ while ensuring structural controllability of the resulting system.

\footnotetext{
${ }^{2}$ The code for this numerical case study is publicly available at https://github.com/odetojsmith/Actuator-Placement-beyond-SC-and-towards-Robustness
} 

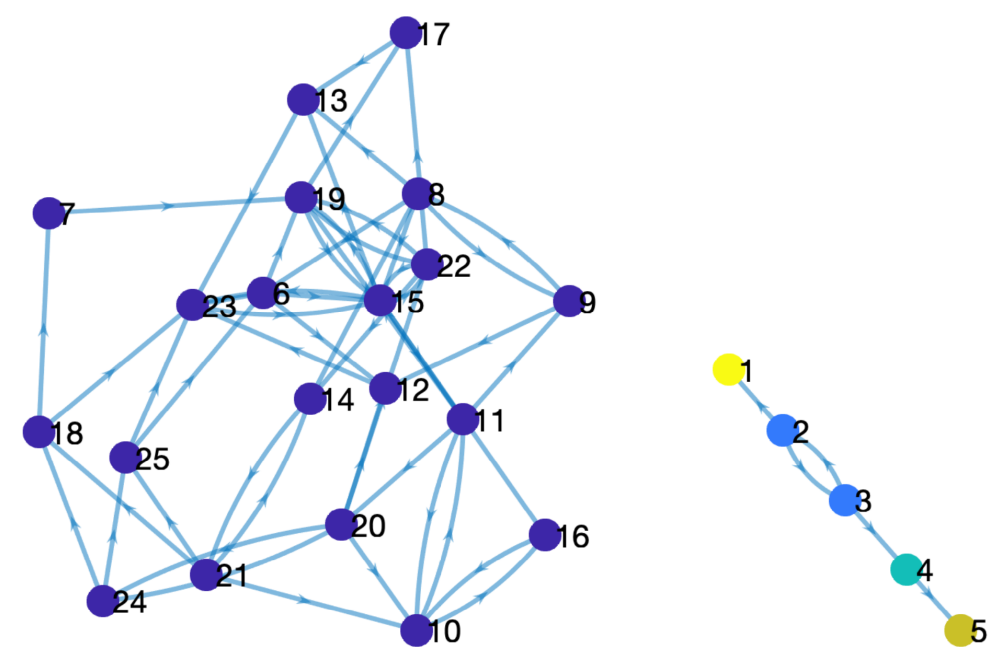

Figure 3: Digraph $G$ under consideration: strongly connected components are marked by different colors

The initial actuator position set derived through Algorithm 2 is $S^{0}=\{16,2\}$, which is straightforward from the observation that, apart from the big strongly connected component containing nodes from 6 to 25 , the node set $\{2,3\}$ is the only strongly connected component without any incoming edges.

The actuator position sets derived through the forward greedy algorithm and the long-horizon greedy algoithm are respectively $S_{\mathrm{f}}=\mathrm{FG}\left(S^{0}, \infty\right)=\{16,2,8,18,11,3,12,5,1\}$ and $S_{\mathrm{If}}=\operatorname{LHFG}\left(S^{0}\right.$, $\infty)=\{16,2,1,13,5,8,24,14,18\}$. The metrics under these two sets are $F_{\epsilon}\left(S_{\mathrm{f}}\right)=7.56 \times 10^{6}$ and $F_{\epsilon}\left(S_{\text {If }}\right)=1.08 \times 10^{5}$. Long horizon greedy provides $98.6 \%$ improvement. The cost we pay for this perforance improvement is the computation time. It is given by 84.7 seconds for deriving $S_{\text {If }}$, whereas 1.3 seconds for deriving $S_{\mathrm{f}}{ }^{3}$ To reduce the computational complexity, we let the horizon of LHFG to be $d_{\mathrm{h}}=3$ and obtain $S_{\text {lf }}^{\prime}=\operatorname{LHFG}\left(S^{0}, 3\right)=\{16,2,25,1,12,5,8,20,24\}$ with $F_{\epsilon}\left(S_{\text {If }}^{\prime}\right)=1.34 \times 10^{6}$. As is discussed before, we do not have any performance guarantees for such modifications. The computation time for $S_{\mathrm{If}}^{\prime}$ is 64.6 seconds.

By checking the structural controllability when a single primary actuator gets offline, we find that the actuators at Node 1 and Node 2 are essential and they require backups. The feasible backup position sets are derived as $\mathcal{G}\left(v_{1}\right)=\left\{v_{1}, v_{3}\right\}$ and $\mathcal{G}\left(v_{2}\right)=\left\{v_{2}\right\}$. Thus, for this system, one can select the backup position set as $\left\{v_{2}, v_{3}\right\}$. Suppose we place primary actuators at $S_{\text {If }}$ and $v_{1}$ goes offline. By activating the backup actuators at $v_{3}$, the metric is $1.07 \times 10^{5}$. In other words, this numerically verifies that the replacement do not jeopardize performance. As a remark, in this example, the minimum number of actuators for structural controllability is $k=3$, less than $K=9$. Even then we see that there are essential actuators, which can result in the loss of structural controllability. Thus, it is necessary to detect such essential actuators and deploy backups.

\footnotetext{
${ }^{3}$ The case study is executed in Matlab R2019b on a computer equipped with $32 \mathrm{~GB}$ RAM and a 2.3GHz Intel Core i9 processor.
} 


\section{Conclusion}

In this paper, we studied the actuator placement problem minimizing a nonsubmodular and a nonsupermodular metric under the constraint of structural controllability. We extended the forward greedy algorithm (FG) to be applicable to arbitrary graphs and we proposed a novel algorithm, LHFG, which was proven to outperform FG. Then, to achieve robustness, we studied the minimal backup actuator placement problem and we showed that it is equivalent to the NP-hard hitting set problem.

Our future work will focus on improving the computational complexity of LHFG and studying backup placement problem in case several primary actuators can go offline, simultaneously.

\section{References}

[1] T. H. Summers, F. L. Cortesi, and J. Lygeros, "On submodularity and controllability in complex dynamical networks," IEEE Trans. on Contr. of Netw. Syst., vol. 3, no. 1, pp. 91$101,2015$.

[2] A. Banerjee and Y. Arkun, "Control configuration design applied to the tennessee eastman plant-wide control problem," Comp. E Chemical Engineering, vol. 19, no. 4, pp. 453-480, 1995.

[3] T. L. Magnanti and R. T. Wong, "Network design and transportation planning: Models and algorithms," Transportation science, vol. 18, no. 1, pp. 1-55, 1984.

[4] J. Chabarek, J. Sommers, P. Barford, C. Estan, D. Tsiang, and S. Wright, "Power awareness in network design and routing," in IEEE INFOCOM 2008-The 27th Conference on Computer Communications. IEEE, 2008, pp. 457-465.

[5] V. Tzoumas, L. Carlone, G. J. Pappas, and A. Jadbabaie, "Lqg control and sensing co-design," IEEE Transactions on Automatic Control, 2020.

[6] T. Summers and M. Kamgarpour, "Performance guarantees for greedy maximization of nonsubmodular controllability metrics," in 2019 18th European Control Conference (ECC), 2019, pp. 2796-2801.

[7] V. Tzoumas, M. A. Rahimian, G. J. Pappas, and A. Jadbabaie, "Minimal actuator placement with bounds on control effort," IEEE Trans. on Contr. of Netw. Syst., vol. 3, no. 1, pp. 67-78, March 2016.

[8] A. A. Bian, J. M. Buhmann, A. Krause, and S. Tschiatschek, "Guarantees for greedy maximization of non-submodular functions with applications," in 34th Int. Conf. on Mach. Lrn., 2017, pp. 498-507.

[9] A. Das and D. Kempe, "Submodular meets spectral: greedy algorithms for subset selection, sparse approximation and dictionary selection," in 28th Int. Conf. on Mach. Lrn., 2011, pp. 1057-1064.

[10] O. Karaca and M. Kamgarpour, "Exploiting weak supermodularity for coalition-proof mechanisms," in CDC. IEEE, 2018, pp. 1118-1123. 
[11] O. Karaca, B. Guo, and M. Kamgarpour, "A comment on performance guarantees of a greedy algorithm for minimizing a supermodular set function on comatroid," EJOR, 2020.

[12] B. Guo, O. Karaca, T. Summers, and M. Kamgarpour, "Actuator placement under structural controllability using forward and reverse greedy algorithms," IEEE Transactions on Automatic Control, 2020.

[13] — - "Actuator placement for optimizing network performance under controllability constraints," in 58th IEEE Conf. on Dec. and Contr., 2019.

[14] C.-T. Lin, "Structural controllability," IEEE Trans. on Aut. Contr., vol. 19, no. 3, pp. 201-208, June 1974.

[15] G. Ramos, A. P. Aguiar, and S. Pequito, "Structural systems theory: an overview of the last 15 years," arXiv preprint arXiv:2008.11223, 2020.

[16] J. Li, X. Chen, S. Pequito, G. J. Pappas, and V. M. Preciado, "On the structural target controllability of undirected networks," IEEE Transactions on Automatic Control, 2020.

[17] J. Jia, H. J. van Waarde, H. L. Trentelman, and M. K. Camlibel, "A unifying framework for strong structural controllability," IEEE Transactions on Automatic Control, vol. 66, no. 1, pp. 391-398, 2020.

[18] Y.-Y. Liu, J.-J. Slotine, and A.-L. Barabási, "Controllability of complex networks," Nature, vol. 473, no. 7346, p. 167, 2011.

[19] C. Commault and J.-M. Dion, "Input addition and leader selection for the controllability of graph-based systems," Automatica, vol. 49, no. 11, pp. 3322-3328, 2013.

[20] A. Clark, L. Bushnell, and R. Poovendran, "On leader selection for performance and controllability in multi-agent systems," in 51st IEEE Conf. on Dec. and Contr., 2012, pp. 86-93.

[21] D. Tihanyi, Y. Lu, O. Karaca, and M. Kamgarpour, "Multi-robot task allocation for safe planning under dynamic uncertainties," arXiv preprint arXiv:2103.01840, 2021.

[22] O. Karaca, D. Tihanyi, and M. Kamgarpour, "Performance guarantees of forward and reverse greedy algorithms for minimizing nonsupermodular nonsubmodular functions on a matroid," arXiv preprint arXiv:2103.01135, 2021.

[23] M. Sviridenko, J. Vondrák, and J. Ward, "Optimal approximation for submodular and supermodular optimization with bounded curvature," 2014.

[24] W. Gao, T. Friedrich, F. Neumann, and C. Hercher, "Randomized greedy algorithms for covering problems," in Proceedings of the Genetic and Evolutionary Computation Conference, 2018, pp. 309-315.

[25] V. Tzoumas, A. Jadbabaie, and G. J. Pappas, "Resilient non-submodular maximization over matroid constraints," arXiv preprint arXiv:1804.01013, 2018.

[26] Q. Hou and A. Clark, "Robust maximization of correlated submodular functions under cardinality and matroid constraints," IEEE Transactions on Automatic Control, 2021. 
[27] Z. Chu and F. Teng, "Short circuit current constrained uc in high ibg-penetrated power systems," arXiv preprint arXiv:2101.04918, 2021.

[28] J. Milošević, A. Teixeira, K. H. Johansson, and H. Sandberg, "Actuator security indices based on perfect undetectability: Computation, robustness, and sensor placement," IEEE Transactions on Automatic Control, vol. 65, no. 9, pp. 3816-3831, 2020.

[29] T. H. Cormen, C. E. Leiserson, R. L. Rivest, and C. Stein, Introduction to algorithms. MIT press, 2009.

[30] L. Lovász and M. Plummer, Matching Theory, ser. North-Holland Math. Studies. Elsevier Science, 1986.

[31] J. Edmonds and R. M. Karp, "Theoretical improvements in algorithmic efficiency for network flow problems," Journal of the ACM (JACM), vol. 19, no. 2, pp. 248-264, 1972.

[32] G. L. Nemhauser, L. A. Wolsey, and M. L. Fisher, "An analysis of approximations for maximizing submodular set functions-I," Math. Prog., vol. 14, no. 1, pp. 265-294, 1978.

[33] J. Edmonds, "Matroids and the greedy algorithm," Math. Prog., vol. 1, no. 1, pp. 127-136, 1971.

[34] E. Angel, E. Bampis, and L. Gourvès, "On the minimum hitting set of bundles problem," Theoretical computer science, vol. 410, no. 45, pp. 4534-4542, 2009.

[35] M. Krivelevich, "Approximate set covering in uniform hypergraphs," Journal of Algorithms, vol. 25, no. 1, pp. 118-143, 1997.

[36] M. El Ouali, H. Fohlin, and A. Srivastav, "A randomised approximation algorithm for the hitting set problem," Theoretical Computer Science, vol. 555, pp. 23-34, 2014. 